\title{
Detection of Asthma and Chronic Obstructive Pulmonary Disease (COPD) With an Electronic Nose (E-Nose) Instrumentation System
}

\author{
N. Sagita, R. A. Putra*, Rahmawati \\ Department of Physics, Faculty of Engineering \\ Universitas Samudra \\ Kota Langsa, Indonesia \\ *rachmad.almi@unsam.ac.id
}

\begin{abstract}
An Electronic Nose (E-Nose) device has been designed to detect asthma and Chronic Obstructive Pulmonary Disease (COPD) through breathing air. This tool consists of hardware and software. E-Nose It consists of a gas sensor that can respond quickly to the elements volatile organic compound (VOC) contained in the respiratory air. The method used is the method Principal Component Analysis (PCA) and Artificial Neural Network (ANN) algorithms backpropagation which is the most precise method of visually classifying types of disease. From the test results using the PCA and ANN methods, it is found that E-Nose can distinguish COPD and asthma with a correlation accuracy of $99.5 \%$ percentage and degree Mean Squared Error (MSE) of 0.005 .
\end{abstract}

Keywords-electronic nose, volatile organic compound, Principal Componen Analysis (PCA), Backpropagation ANN, COPD and asthma

\section{INTRODUCTION}

There are currently several respiratory diseases that are a global problem. These include Chronic Obstructive Pulmonary Disease (COPD) and Asthma [1,2]. Currently there are several methods to diagnose pulmonary disease, among others, anamnesis, physical examination, X-ray, computerized aided tomography (CT) scan, phlegm cell examination and blood test [3]. This method takes a long time and requires a lot of money, causing people to be reluctant to have their health checked. Therefore, we need a new method which is more accurate and less expansive. For example, namely by developing an Electronic Nose (E-Nose) to detect disease through breathing air [4].

Volatile Organic Compound issued by the body contained in the respiratory air has a special pattern according to genetic or environmental factors, so it can be used as a biomarker in diagnosing a disease derived from the lungs, such as Chronic Obstructive Pulmonary Disease (COPD) and asthma [5]. ENose is one of the instruments to transform the aroma into a sensor response, then the sensor response will be analyzed by utilizing the Artificial Neural Network (JST) method [6].

\author{
M. Yakob \\ Department of Physics Education, Faculty of Teaching and \\ Education \\ Universitas Samudra \\ Kota Langsa, Indonesia
}

Based on the problems discussed above, it can be assumed that E-Nose has the ability to detect a disease through the respiratory air by detecting the real-time pattern of VOC produced when a person breathes. The pattern of VOC can be used as a biomarker in classifying COPD disease and asthma [7]. In this research will be discussed how to design an E-Nose that is able to detect diseases of COPD and asthma through the respiratory air using the method of Principal Component Analysis (PCA) and Neural Network Mock (JST) algorithm backpropagation.

\section{METHODS}

There are two main methods in this research:

\section{A. Design Stage}

At this stage, two designs will be carried out, namely hardware design and Electronic Nose software design.

1) Hardware design: Design hardware in this research is making tool design E-Nose to detect Chronic Obstructive Pulmonary Disease (COPD) and Asthma which consists of Arduino Atmega, six MQ sensors namely MQ 2, MQ 4, MQ 7, MQ 5, MQ 9, and MQ 135, LCD, heater, four fans, dimmer, breadboard, temperature sensor, and jumper cable. Figure 1 is the Electronic Nose hardware design.

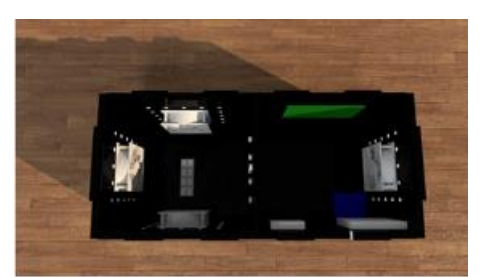

(a)

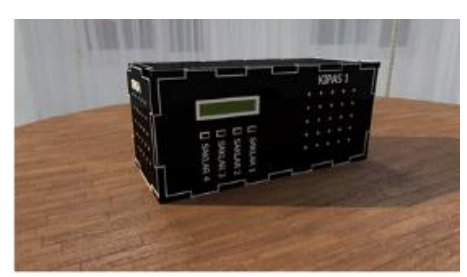

(b)

Fig. 1. (a) Prototype E-Nose detectors of COPD and Asthma appear outward, (b) Prototype E- Nose detectors of COPD and Asthma appear deep. 
In hardware design, there is a schematic of the electronic circuit from the Electronic Nose system that can be seen in the following figure 2 :

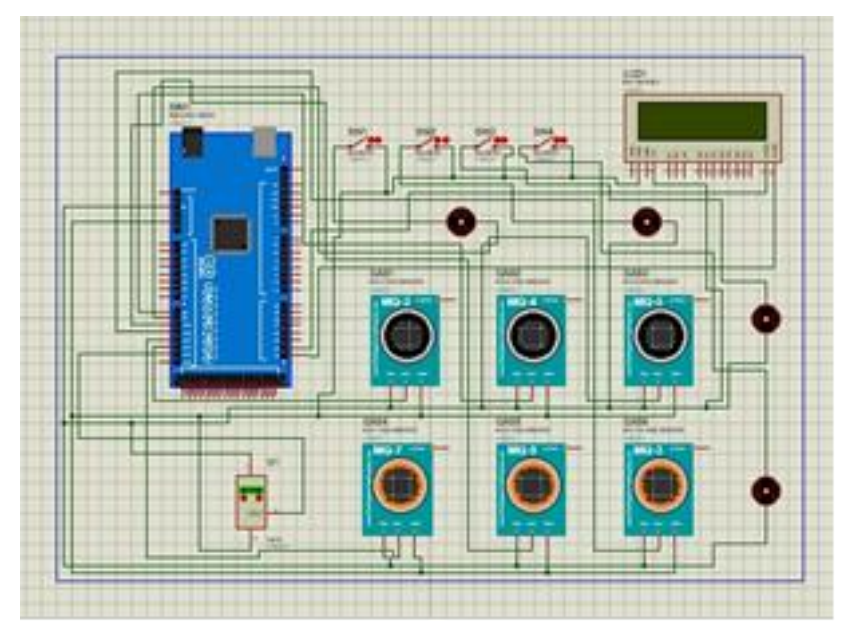

Fig. 2. Circuit schematic.

2) Software design: In carrying out the system design ENose requires a controller in the form of a program. A system design E-Nose 2 types of programs are used, namely, the control program using software Arduino and as data logger use software MATLAB R2010a. MATLAB R2010a software is used to display data in graphical form on computer devices. The data graph displayed is the voltage value received by the microcontroller from the sensor. Data in the form of voltage values will then be stored in the Microsoft Excel application where the analysis will be carried out to see the pattern of the breath odor of a person who is the sample data. See figure 3 below.

\section{B. Sampling Stage}

At the sampling stage in this research, it was designed based on the flowchart below:

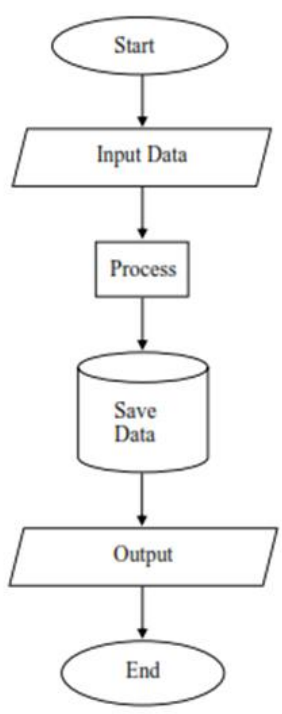

(a)

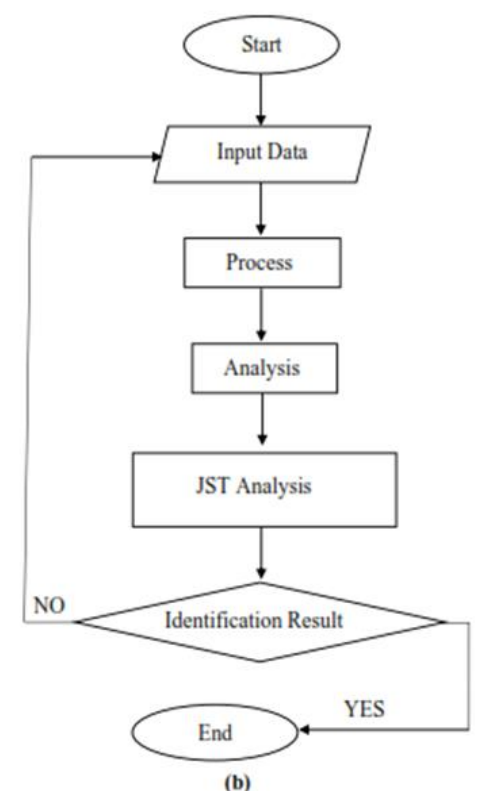

(b)
Fig. 3. a) Flowchart train, (b) Flowchart testing.

Sampling in this research was carried out by illustrating the patient to breathe normally using an oxygen mask connected to an Electronic Nose device. The time it takes the patient to breathe is around three minutes. The output data from the readings are recorded using the Data Logger User Interface (GUI). Meanwhile, data processing will be carried out using the Main Component Analysis (PCA) method. Respiratory air that is used as a sample is the respiratory air of patients who have indications of COPD or Asthma.

\section{RESUlTS AND DISCUSSION}

\section{A. Electronic Nose (E-Nose) Instrumentation}

E-Nose is connected to the computer (PC). The analog signal read by the sensor is visualized in $2 \mathrm{D}$ graph form as in Figure 4 below. The visualization data that is formed comes from quantitative data on the voltage on the sensor which is influenced by the organic compounds (volatile organic compounds) present in the breathing air. The visualization data will then be stored on a computer (PC). The result of data recording stored on the computer will form an $\mathrm{N} \times \mathrm{M}$ matrix, where $\mathrm{N}$ is the number of data samples, while $\mathrm{M}$ is the number of sensors used. 


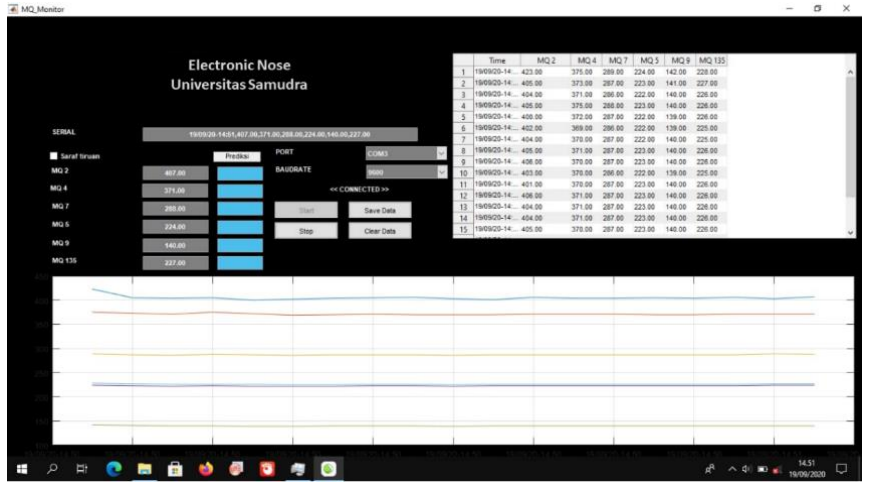

Fig. 4. GUI logger data visualization of signals E-Nose.

\section{B. Data Sampling Analysis}

The first data to be analyzed is zero data. Zero data is blank data from E-Nose without breath samples. The zero data collection aims to see the difference in the response of the sensor without samples and with breath samples containing volatile organic compound (VOC) compounds. After taking zero data, the next data analyzed were COPD and asthma respiratory samples. Figure 5 is the result of analysis using the PCA method.

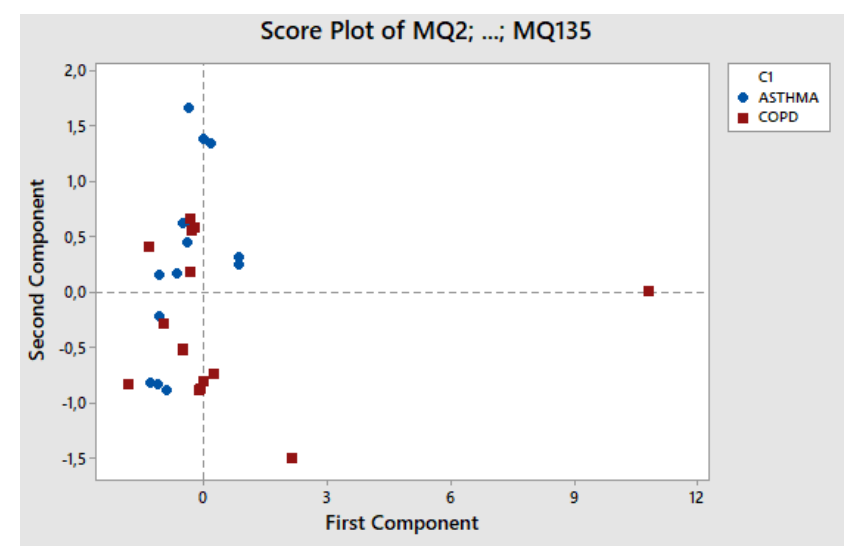

Fig. 5. Distribution of COPD and asthma sample data.

Figure 5 shows the distribution of data on COPD and asthma samples. The results of the PCA analysis showed that each sample was scattered close together, there were several samples intersecting between copd with asthma samples, this is because there are similar volatile gases between the two types of samples. If created in the grouping plot score, it can be seen the difference between COPD and asthma samples as shown in Figure 6.

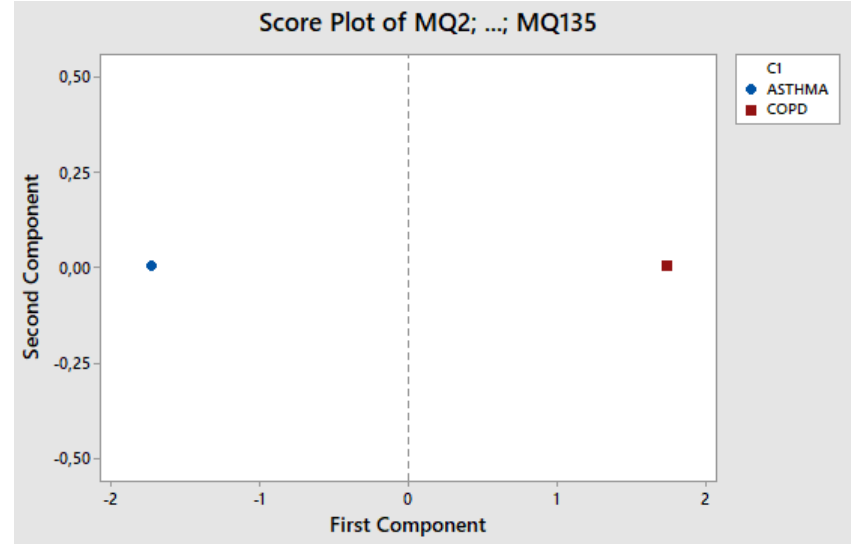

Fig. 6. Grouping COPD and asthma sample data.

Figure 6 explains that COPD and asthma samples are separate from each other which indicates that both types of samples can be well identified. There are certain volatile gas indicators or biomarkers in a persons respiratory air that can indicate a disease. Here Table 1 is a volatile gas biomarker for COPD disease and asthma.

TABLE I. VOLATILE BIOMARKERS IN HUMANS

\begin{tabular}{|l|l|l|l|}
\hline No & Volatile Biomarkers & Disease & Reference \\
\hline 1 & Hydrogen Peroxide & CPOD & {$[7-10]$} \\
\hline & Ethane & & \\
\hline & 8-Isoprostane & & \\
\hline & Leukotriene B4 & & \\
\hline & Nitric Oxide & & \\
\hline & Nitrotyrosine & & \\
\hline & Alkanes & & \\
\hline & Short Chain HC & Asthma & {$[7,8]$} \\
\hline & Hydrogen Peroxide & & \\
\hline & Alkanes & & \\
\hline & Short Chain HC & & \\
\hline & Isoprene & & \\
\hline & Leukotriene B4 & & \\
\hline & Nitric Oxide & & \\
\hline & Pentane & & \\
\hline & 8-Isoprostane & \\
\hline
\end{tabular}

Biomarkers are the ones that can be used to detect COPD and asthma diseases through breathing air. Here are the PCA results that show the projected results of loading plots against the magnitude of the response of each sensor. 


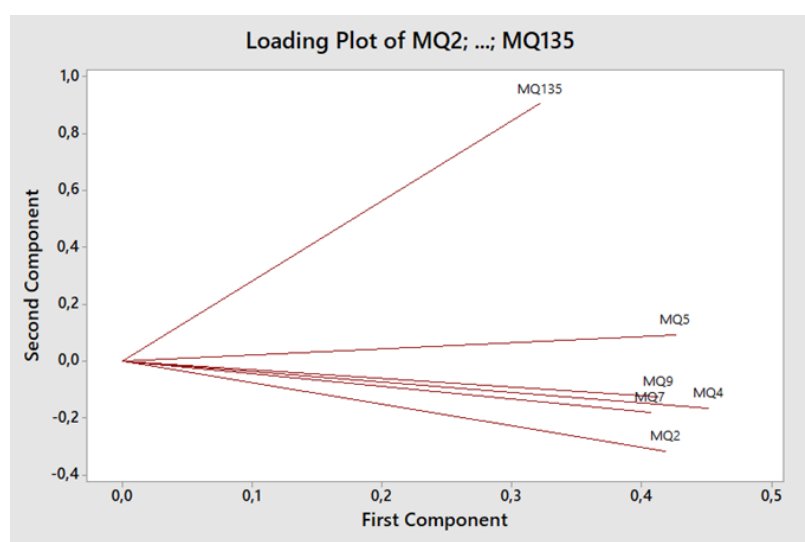

Fig. 7. Loading plot E-Nose system against sample.

Figure 7 is a loading plot that shows the difference in each sensor's response to the sample. From Figure 7, it can be ensured that volatile gases from the respiratory air of COPD and asthma tested can be responded well by all sensors. The different line lengths on the loading plot indicate the difference in response of each sensor to the sample. The longer the line is formed, the greater the sensor's contribution in identifying a sample it detects. There are five sensors that have the longest line namely MQ 4, MQ 5, MQ 2, MQ 9 and MQ 7. These sensors contribute greatly to the sample differentiator parameters. While the MQ 135 sensor is the least sensor in sample classification. This is because the MQ 135 sensor can only detect a few of the biomarkers of COPD and asthma.

From the results of tests that have been conducted using the PCA method shows good classification results between COPD and asthma. Figure 8 and Figure 9 are patterns for COPD and asthma.

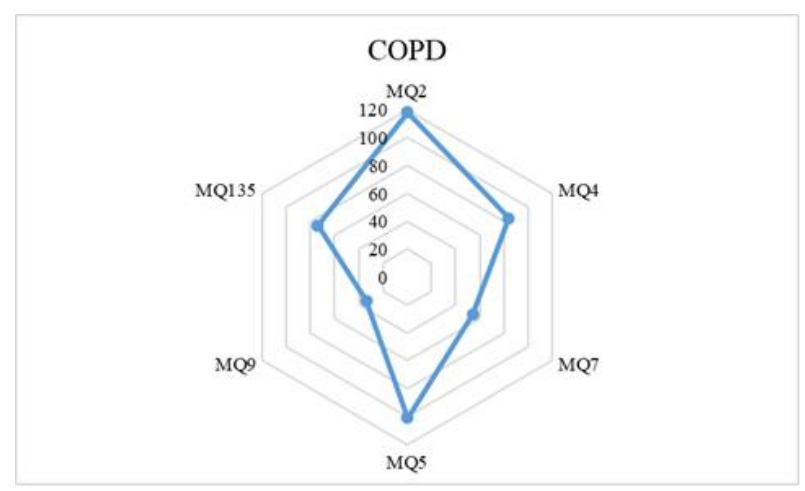

Fig. 8. COPD disease radar plot.

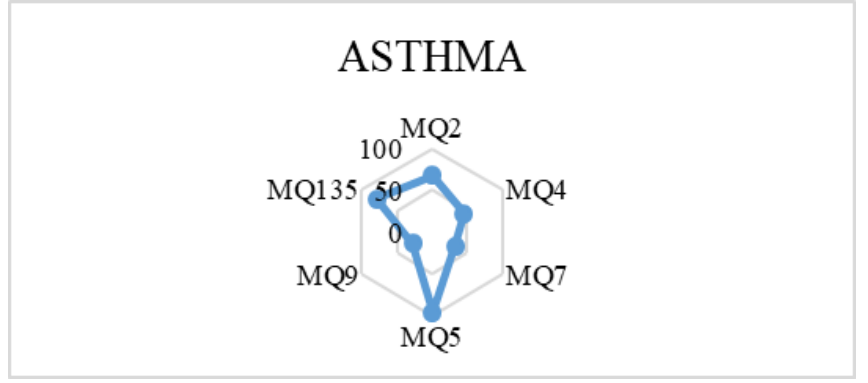

Fig. 9. Asthma radar plot.

After the analysis process using the PCA method is completed then continued by using the JST method. Figure 10 is the result of an analysis using the JST method of the backpropagation algorithm.

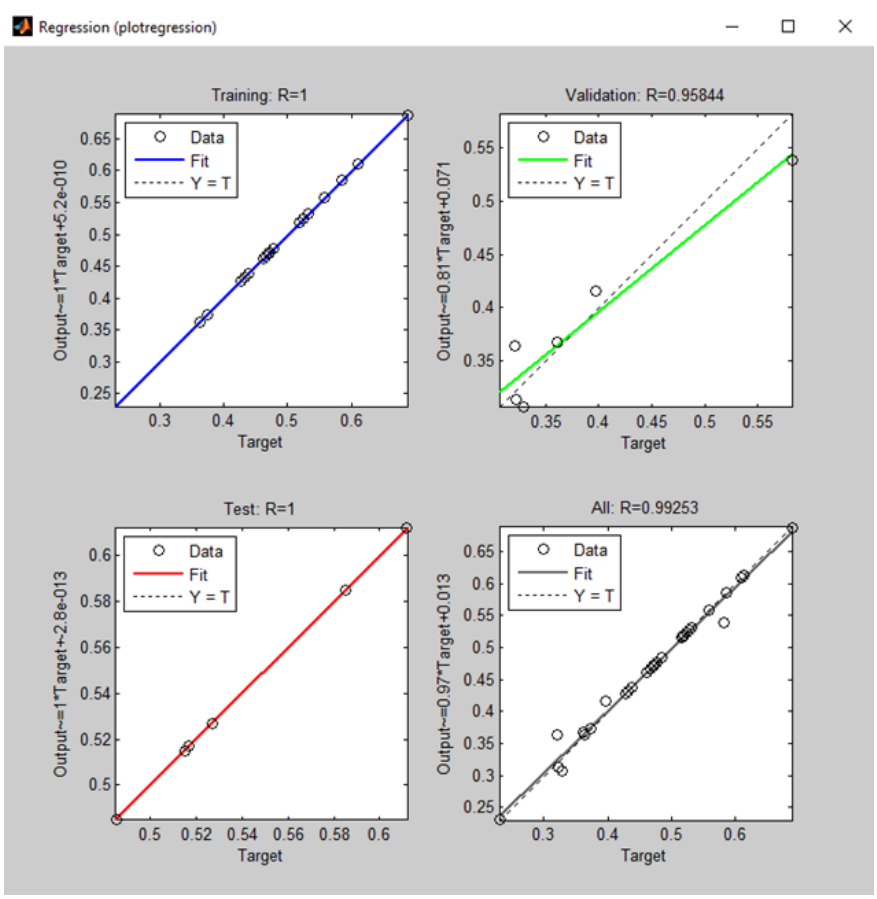

Fig. 10. Plot regression of COPD and asthma disease.

Figure 10 shows that the JST method of backpropagation algorithm has been able to predict COPD and asthma disease well and result in the accuracy of the percentage correlation between the target and output by $99.5 \%$ with MSE of 0.005 . The accuracy of the correlation percentage and MSE is obtained by looking at the results of the regression plot and using equations $(1-\alpha) 100 \%$ [11].

\section{CONCLUSION}

Based on the results of the study, it can be concluded that E-Nose can distinguish between COPD and asthma using biomarkers found in the respiratory air using the PCA method and produce accuracy of the percentage correlation between the target and output by $99.5 \%$ with MSE of 0.005 using the JST method of backpropagation algorithm. 


\section{ACKNOWLEDGMENT}

Thank you to Universitas Samudra for funding this research through its 2020 flagship applied research grant program (PTU).

\section{REFERENCES}

[1] P.W. Jones, "Global Strategy for the Diagnosis , Management, and Prevention of Chronic Obstructive Pulmonary Disease," Pulm. Perspect., vol. 187, no. 4, pp. 347-365, 2020.

[2] R. Lozano, "Global and regional mortality from 235 causes of death for 20 age groups in 1990 and 2010: a systematic analysis for the Global Burden of Disease Study 2010," Articles, vol. 380, pp. 2095-2128, 2010.

[3] I.D. Kurniati, Buku Ajar Ilmu Penyakit Dalam. 2015.

[4] S. Carlos, J.P. Santos, and J. Lozano, "Use of Electronic Noses for Diagnosis of Digestive and Respiratory Diseases through the Breath," Biosensors, vol. 9, no. 35, pp. 1-20, 2019.
[5] P. Montuschi, N. Mores, C. Mondino, and P.J. Barnes, "The Electronic Nose in Respiratory Medicine," Respiration, vol. 85, pp. 72-84, 2013.

[6] I.N. Rahmani, D. Lelono, and K. Triyana, "Klasifikasi Kakao Berbasis E-Nose Dengan Metode Neuro Fuzzy," Indones. J. Electron. Instrum. Syst., vol. 8, no. 1, pp. 49-60, 2018.

[7] M. Yakob, D. Mustika, I.R. Nila, and R.A. Putra, "Design of E-nose as an Instrument Identification of Diseases Through the Respiratory Tract Design of E-nose as an Instrument Identification of Diseases Through the Respiratory Tract," 4th Int. Conf. Appl. Phys. Mater. Appl., vol. 1428, no. 012062, pp. 1-7, 2020.

[8] A.D. Wilson, "Advances in electronic-nose technologies for the detection of volatile biomarker metabolites in the human breath," Metabolites, vol. 5, no. 1, pp. 140-163, 2015.

[9] N. Maulidiyah and M. Amin, "Biomarker Pernapasan pada Penyakit Paru,” J. Respirasi, vol. 1, no. 2, p. 67, 2019.

[10] J.J.B.N. Van Berkel, "A profile of volatile organic compounds in breath discriminates COPD patients from controls," Respir. Med., vol. 104, no. 4, pp. 557-563, 2010.

[11] S. Azwar, "Signifikan Atau Sangat Signifikan ?," Bul. Psikol., vol. 13, no. I, pp. 38-44, 2005. 